\title{
A study on vague graphs
}

\author{
Hossein Rashmanlou', Sovan Samanta2 ${ }^{2}$, Madhumangal Pal $^{3}$ and R. A. Borzooei ${ }^{4}$
}

\author{
*Correspondence: \\ ssamantavu@gmail.com \\ ${ }^{2}$ Department \\ of Mathematics, Indian \\ Institute of Information \\ Technology, Nagpur 440006, \\ India \\ Full list of author information \\ is available at the end of the \\ article
}

\begin{abstract}
The main purpose of this paper is to introduce the notion of vague h-morphism on vague graphs and regular vague graphs. The action of vague h-morphism on vague strong regular graphs are studied. Some elegant results on weak and co weak isomorphism are derived. Also, $\mu$-complement of highly irregular vague graphs are defined.
\end{abstract}

Keywords: Vague graph, Vague h-morphism, Highly irregular vague graph

\section{Background}

Gau and Buehrer (1993) proposed the concept of vague set in 1993, by replacing the value of an element in a set with a subinterval of $[0,1]$. Namely, a true-membership function $t_{v}(x)$ and a false membership function $f_{v}(x)$ are used to describe the boundaries of the membership degree. The initial definition given by Kauffman (1973) of a fuzzy graph was based the fuzzy relation proposed by Zadeh. Later Rosenfeld (1975) introduced the fuzzy analogue of several basic graph-theoretic concepts. After that, Mordeson and Nair (2001) defined the concept of complement of fuzzy graph and studied some operations on fuzzy graphs. Sunitha and Vijayakumar (2002) studied some properties of complement on fuzzy graphs. Many classifications of fuzzy graphs can be found in Boorzooei et al. (2016a, b), Rashmanlou and Pal (2013a, b), Rashmanlou et al. (2015a, b, c), Pramanik et al. (2016a, b), Samanta and Pal (2011b, 2012b, 2015, 2016) and Samanta et al. (2014c, d). Recently, Samanta and Pal (2011a, 2012a, 2013, 2014a, 2015) and Samanta et al. (2014b) defined different types of fuzzy graphs and established some important properties. To extent the theory of fuzzy graphs, Akram et al. (2014) introduced vague hypergraphs. After that, Ramakrishna (2009) introduced the concept of vague graphs and studied some of their properties.

In this paper, we introduce the notion of vague h-morphism on vague graphs and study the action of vague h-morphism on vague strong regular graphs. We derive some elegant results on weak and co weak isomorphism. Also, we define $\mu$-complement of highly irregular vague graphs.

\section{Preliminaries}

By a graph $G^{*}=(V, E)$, we mean a non-trivial, finite connected and undirected graph without loops or multiple edges. A fuzzy graph $G=(\sigma, \mu)$ is a pair of functions $\sigma: V \rightarrow[0,1]$ and $\mu: V \times V \rightarrow[0,1]$ with $\mu(u, v) \leq \sigma(u) \wedge \sigma(v)$, for all $u, v \in V$, where $V$ is a finite non-empty set and $\wedge$ denote minimum.

(c) 2016 The Author(s). This article is distributed under the terms of the Creative Commons Attribution 4.0 International License (http://creativecommons.org/licenses/by/4.0/), which permits unrestricted use, distribution, and reproduction in any medium, provided you give appropriate credit to the original author(s) and the source, provide a link to the Creative Commons license, and indicate if changes were made. 
Definition 1 (Gau and Buehrer 1993) A vague set $A$ on a set $X$ is a pair $\left(t_{A} ; f_{A}\right)$ where $t_{A}$ and $f_{A}$ are real valued functions defined on $X \rightarrow[0,1]$, such that $t_{A}(x)+f_{A}(x)<1$ for all $x \in X$. The interval $\left[t_{A}(x), 1-f_{A}(x)\right]$ is called the vague value of $x$ in $A$.

In the above definition, $t_{A}(x)$ is considered as the lower bound for degree of membership of $x$ in $A$ and $f_{A}(x)$ is the lower bound for negative of membership of $x$ in $A$. So, the degree of membership of $x$ in the vague set $A$ is characterized by the interval $\left[t_{A}(x), 1-f_{A}(x)\right]$

The definition of intuitionistic fuzzy graphs is follows. Let $V$ be a non-empty set. An intuitionistic fuzzy set (IFS) in $V$ is represented by $(V, \mu, v)$, where $\mu: V \rightarrow[0,1]$ and $v: V \rightarrow[0,1]$ are membership function and non-membership function respectively such that $0 \leq \mu(x)+v(x) \leq 1$ for all $x \in V$. Though intuitionistic fuzzy sets and vague sets look similar, analytically vague sets are more appropriate when representing vague data. The difference between them is discussed below.

The membership interval of an element $x$ for vague set $A$ is $\left[t_{A}(x), 1-f_{A}(x)\right]$. But, the membership value for an element $y$ in an intuitionistic fuzzy set $B$ is $\left\langle\mu_{B}(y), v_{B}(y)\right\rangle$. Here, the semantics of $t_{A}$ is the same as with $A$ and $\mu_{B}$ is the same as with $B$. However, the boundary is able to indicate the possible existence of a data value. This difference gives rise to a simpler but meaningful graphical view of data sets (see Fig. 1). It can be seen that, the shaded part formed by the boundary in a given Vague Set naturally represents the possible existence of data. Thus, this "hesitation region" corresponds to the intuition of representing vague data. We will see more benefits of using vague membership intervals in capturing data semantics in subsequent sections.

Let $X$ and $Y$ be ordinary finite non-empty sets. We call a vague relation to be a vague subset of $X \times Y$, that is an expression $R$ defined by

$$
R=\left\{\left\langle(x, y), t_{R}(x, y), f_{R}(x, y)\right\rangle \mid x \in X, y \in Y\right\}
$$

where $t_{R}: X \times Y \rightarrow[0,1], f_{R}: X \times Y \rightarrow[0,1]$ which satisfies the condition $0 \leq$ $t_{R}(x, y)+f_{R}(x, y) \leq 1$, for all $(x, y) \in X \times Y$. A vague relation $R$ on $X$ is called reflexive if $t_{R}(x, x)=1$ and $f_{R}(x, x)=0$ for all $x \in X$. A vague relation $R$ is symmetric if $t_{R}(x, y)=t_{R}(y, x)$ and $f_{R}(x, y)=f_{R}(y, x)$, for all $x, y \in X$.

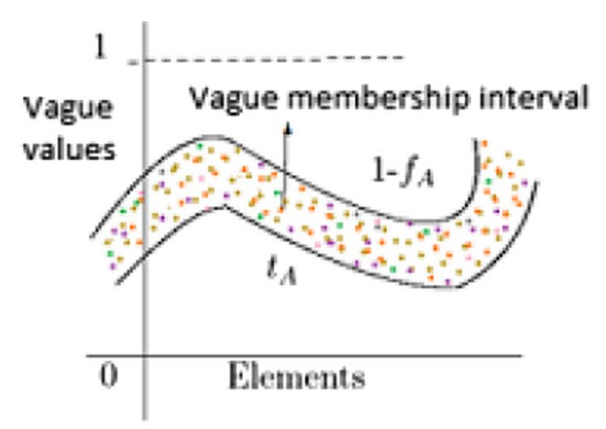

a

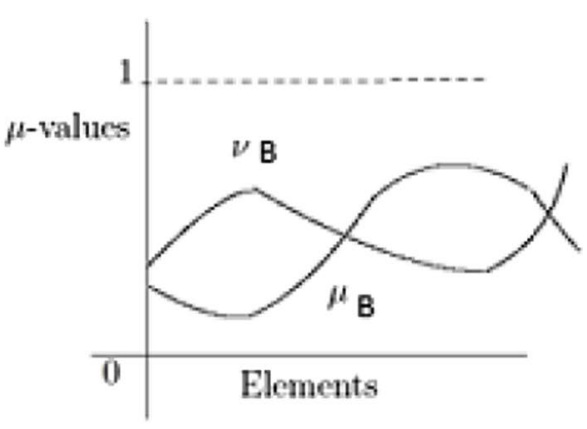

b

Fig. 1 Comparison between $\mathbf{a}$ vague sets and $\mathbf{b}$ intutionistic fuzzy sets 
Definition 2 Let $G^{*}=(V, E)$ be a crisp graph. A pair $G=(A, B)$ is called a vague graph on a crisp graph $G^{*}=(V, E)$, where $A=\left(t_{A}, f_{A}\right)$ is a vague set on $V$ and $B=\left(t_{B}, f_{B}\right)$ is a vague set on $E \subseteq V \times V$ such that $t_{B}(x y) \leq \min \left(t_{A}(x), t_{A}(y)\right)$ and $f_{B}(x y) \geq \max$ $\left(f_{A}(x), f_{A}(y)\right)$ for each edge $x y \in E$. A vague graph $G$ is called strong if $t_{B}(x y)=\min \left(t_{A}(x), t_{A}(y)\right)$ and $f_{B}(x y)=\max \left(f_{A}(x), f_{A}(y)\right)$ for all $x, y \in V$.

Definition 3 The vague graph $G$ is said to be regular if $\sum_{v_{j}, v_{i} \neq v_{j}} t_{B}\left(v_{i} v_{j}\right)=$ constant and $\sum_{v_{j}, v_{i} \neq v_{j}} f_{B}\left(v_{i} v_{j}\right)=$ constant, for all $v_{i} \in V$. Moreover, it is called strong regular if

1. $t_{B}\left(v_{i} v_{j}\right)=\min \left\{t_{A}\left(v_{i}\right), t_{A}\left(v_{j}\right)\right\}$ and $f_{B}\left(v_{i} v_{j}\right)=\max \left\{f_{A}\left(v_{i}\right), f_{A}\left(v_{j}\right)\right\}$.

2. $\sum_{v_{j}, v_{i \neq v_{j}}} t_{B}\left(v_{i} v_{j}\right)=$ constant and $\sum_{v_{j}, v_{i \neq v_{j}}} f_{B}\left(v_{i} v_{j}\right)=$ constant.

Definition 4 The complement of a vague graph $G=(A, B)$ is a vague graph $\bar{G}=(\bar{A}, \bar{B})$ where $\bar{A}=A$ and $\bar{B}$ is described as follows. The true and false membership values for edges of $\bar{G}$ are given below.

$$
\overline{t_{B}}(u v)=t_{A}(u) \wedge t_{A}(v)-t_{B}(u v) \text { and } \overline{f_{B}}(u v)=f_{B}(u v)-f_{A}(u) \vee f_{A}(v) \text {, for all } u, v \in V \text {. }
$$

Definition 5 Let $G_{1}$ and $G_{2}$ be two vague graphs.

1. A homomorphism $h$ from $G_{1}$ to $G_{2}$ is a mapping $h: V_{1} \rightarrow V_{2}$ which satisfies the following conditions:

(a) $t_{A_{1}}\left(x_{1}\right) \leq t_{A_{2}}\left(h\left(x_{1}\right)\right), f_{A_{1}}\left(x_{1}\right) \geq f_{A_{2}}\left(h\left(x_{1}\right)\right)$,

(b) $t_{B_{1}}\left(x_{1} y_{1}\right) \leq t_{B_{2}}\left(h\left(x_{1}\right) h\left(y_{1}\right)\right), f_{B_{1}}\left(x_{1} y_{1}\right) \geq f_{B_{2}}\left(h\left(x_{1}\right) h\left(y_{1}\right)\right)$ for all $x_{1} \in V_{1}, x_{1} y_{1} \in E_{1}$.

2. An isomorphism $h$ from $G_{1}$ to $G_{2}$ is a bijective mapping $h: V_{1} \rightarrow V_{2}$ which satisfies the following conditions:

(c) $t_{A_{1}}\left(x_{1}\right)=t_{A_{2}}\left(h\left(x_{1}\right)\right), f_{A_{1}}\left(x_{1}\right)=f_{A_{2}}\left(h\left(x_{1}\right)\right)$,

(d) $t_{B_{1}}\left(x_{1} y_{1}\right)=t_{B_{2}}\left(h\left(x_{1}\right) h\left(y_{1}\right)\right), f_{B_{1}}\left(x_{1} y_{1}\right)=f_{B_{2}}\left(h\left(x_{1}\right) h\left(y_{1}\right)\right)$, for all $x_{1} \in V_{1}, x_{1} y_{1} \in E_{1}$.

3. A weak isomorphism $h$ from $G_{1}$ to $G_{2}$ is a bijective mapping $h: V_{1} \rightarrow V_{2}$ which satisfies the following conditions:

(e) $h$ is homomorphism,

(f) $t_{A_{1}}\left(x_{1}\right)=t_{A_{2}}\left(h\left(x_{1}\right)\right), f_{A_{1}}\left(x_{1}\right)=f_{A_{2}}\left(h\left(x_{1}\right)\right)$ for all $x_{1} \in V_{1}$. Thus a weak isomorphism preserves the weights of the nodes but not necessarily the weights of the arcs.

4. A co weak isomorphism $h$ from $G_{1}$ to $G_{2}$ is a bijective mapping $h: V_{1} \rightarrow V_{2}$ which satisfies:

(g) $h$ is homomorphism,

(h) $t_{B_{1}}\left(x_{1} y_{1}\right)=t_{B_{2}}\left(h\left(x_{1}\right) h\left(y_{1}\right)\right), f_{B_{1}}\left(x_{1} y_{1}\right)=f_{B_{2}}\left(h\left(x_{1}\right) h\left(y_{1}\right)\right)$, for all $x_{1} y_{1} \in E_{1}$. 


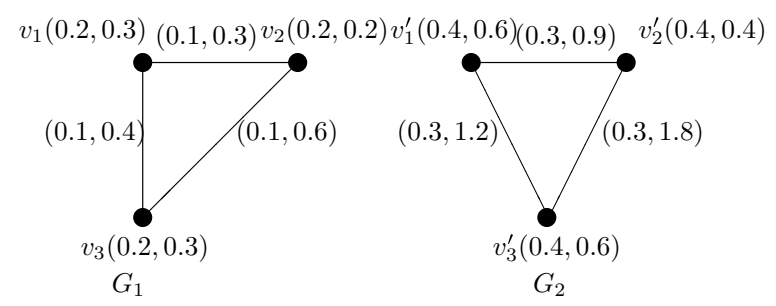

Fig. 2 h-Morphism of vague graphs $G_{1}$ and $G_{2}$

Definition 6 Let $G=(A, B)$ be a vague graph on $G^{*}$.

1. The open degree of a vertex $u$ is defined as $\operatorname{deg}(u)=\left(d_{t}(u), d_{f}(u)\right)$, where

$$
\begin{aligned}
d_{t}(u) & =\sum_{\substack{u \neq v \\
v \in V}} t_{B}(u v) \text { and } \\
d_{f}(u) & =\sum_{\substack{u \neq v \\
v \in V}} f_{B}(u v) .
\end{aligned}
$$

2. The order of $G$ is defined and denoted as

$$
O(G)=\left(\sum_{u \in V} t_{A}(u), \sum_{u \in V} f_{A}(u)\right) .
$$

3. The size of $G$ is defined as $S(G)=\left(S_{t}(G), S_{f}(G)\right)=\left(\sum_{\substack{u \neq v \\ u, v \in V}} t_{B}(u v), \sum_{\substack{u \neq v \\ u, v \in V}} f_{B}(u v)\right)$.

\section{Regularity on isomorphic vague graph}

In this section, we introduce the notion of vague $h$-morphism on vague graphs and regular vague graph. We derive some elegant results on weak and co weak isomorphisms.

Definition 7 Let $G_{1}$ and $G_{2}$ be two vague graphs on $\left(V_{1}, E_{1}\right)$ and $\left(V_{2}, E_{2}\right)$, respectively. A bijective function $h: V_{1} \rightarrow V_{2}$ is called vague morphism or vague $h$-morphism if there exists positive numbers $k_{1}$ and $k_{2}$ such that $(i) t_{A_{2}}(h(u))=k_{1} t_{A_{1}}(u)$ and $f_{A_{2}}(h(u))=k_{1} f_{A_{1}}(u)$, for all $u \in V_{1},(i i) t_{B_{2}}(h(u) h(v))=k_{2} t_{B_{1}}(u v)$ and $f_{B_{2}}(h(u) h(v))=k_{2} f_{B_{1}}(u v)$, for all $u v \in E_{1}$. In such a case, $h$ will be called a $\left(k_{1}, k_{2}\right)$ vague $h$-morphism from $G_{1}$ to $G_{2}$. If $k_{1}=k_{2}=k$, we call $h$, a vague $\mathrm{k}$-morphism. When $k=1$ we obtain usual vague morphism.

Example 8 Consider two vague graphs $G_{1}$ and $G_{2}$ defined as follows (see Fig. 2). Here, there is a vague $h$-morphism such that $h\left(v_{1}\right)=v_{1}^{\prime}, h\left(v_{2}\right)=v_{2}^{\prime}, h\left(v_{3}\right)=v_{3}^{\prime}, k_{1}=2$, and $k_{2}=3$.

Theorem 9 The relation h-morphism is an equivalence relation in the collection of vague graphs.

Proof Consider the collection of vague graphs. Define the relation $G_{1} \approx G_{2}$ if there exists a $\left(k_{1}, k_{2}\right)$ h-morphism from $G_{1}$ to $G_{2}$ where both $K_{1}$ and $K_{2}$ are non-zero. Consider 
the identity morphism from $G_{1}$ to $G_{1}$. It is a $(1,1)$ morphism from $G_{1}$ to $G_{1}$ and hence $\approx$ is reflexive.

Let $G_{1} \approx G_{2}$. Then there exists a $\left(k_{1}, k_{2}\right)$ morphism from $G_{1}$ to $G_{2}$, for some nonzero $k_{1}$ and $k_{2}$. Therefore $t_{A_{2}}(h(u))=k_{1} t_{A_{1}}(u), f_{A_{2}}(h(u))=k_{1} f_{A_{1}}(u)$, for all $u \in V_{1}$ and $t_{B_{2}}(h(u) h(v))=k_{2} t_{B_{1}}(u v)$ and $f_{B_{2}}(h(u) h(v))=k_{2} f_{B_{1}}(u v)$, for all $u v \in E_{1}$. Consider $h^{-1}: G_{2} \rightarrow G_{1}$. Let $x, y \in V_{2}$. Since $h^{-1}$ is bijective, $x=h(u), y=h(v)$, for some $u, v \in V_{2} . \quad$ Now, $\quad t_{A_{1}}\left(h^{-1}(x)\right)=t_{A_{1}}\left(h^{-1}(h(u))\right)=t_{A_{1}}(u)=\frac{1}{k_{1}} t_{A_{2}}(h(u))=\frac{1}{k_{1}} t_{A_{2}}(x)$. $f_{A_{1}}\left(h^{-1}(x)\right)=f_{A_{1}}\left(h^{-1}(h(u))\right)=f_{A_{1}}(u)=\frac{1}{k_{1}} f_{A_{2}}(h(u))=\frac{1}{k_{1}} f_{A_{2}}(x) . \quad t_{B_{1}}\left(h^{-1}(x) h^{-1}(y)\right)=$ $t_{B_{1}}\left(h^{-1}(h(u)) h^{-1}(h(v))\right)=t_{B_{1}}(u v)=\frac{1}{k_{2}} t_{B_{2}}(h(u) h(v))=\frac{1}{k_{2}} t_{B_{2}}(x y), t_{B_{1}}\left(h^{-1}(x) h^{-1}(y)\right)=$ $t_{B_{1}}\left(h^{-1}(h(u)) h^{-1}(h(v))\right)=t_{B_{1}}(u v)=\frac{1}{k_{2}} t_{B_{2}}(h(u) h(v))=\frac{1}{k_{2}} t_{B_{2}}(x y), f_{B_{1}}\left(h^{-1}(x) h^{-1}(y)\right)=$ $f_{B_{1}}\left(h^{-1}(h(u)) h^{-1}(h(v))\right)=f_{B_{1}}(u v)=\frac{1}{k_{2}} f_{B_{2}}(h(u) h(v))=\frac{1}{k_{2}} f_{B_{2}}(x y)$. Thus there exists $\left(\frac{1}{k_{1}}, \frac{1}{k_{2}}\right)$ morphism from $G_{2}$ to $G_{1}$. Therefore, $G_{2} \approx G_{1}$ and hence $\approx$ is symmetric.

Let $G_{1} \approx G_{2}$ and $G_{2} \approx G_{3}$. Then there exists a $\left(k_{1}, k_{2}\right)$ morphism from $G_{1}$ to $G_{2}$ say $h$ for some non-zero $k_{1}$ and $k_{2}$ and there exists $\left(k_{3}, k_{4}\right)$ morphism from $G_{2}$ to $G_{3}$ say $g$ for some non-zero $k_{3}$ and $k_{4}$. So, $t_{A_{3}}(g(x))=k_{3} t_{A_{2}}(x)$ and $f_{A_{3}}(g(x))=k_{3} f_{A_{2}}(x)$, for all $x \in V_{2}$ and $t_{B_{3}}(g(x) g(y))=k_{4} t_{B_{2}}(x y)$ and $f_{B_{3}}(g(x) g(y))=k_{4} f_{B_{2}}(x y)$, for all $x y \in E_{2}$. Let $f=g \circ h: G_{1} \rightarrow G_{3}$. Now,

$$
\begin{aligned}
t_{A_{3}}(f(u))=t_{A_{3}}((g \circ h)(u)) & =t_{A_{3}}(g(h(u))) \\
& =k_{3} t_{A_{3}}(h(u)) \\
& =k_{3} k_{1} t_{A_{1}}(u) \\
f_{A_{3}}(f(u))=f_{A_{3}}((g \circ h)(u)) & =f_{A_{3}}(g(h(u))) \\
& =k_{3} f_{A_{3}}(h(u)) \\
& =k_{3} k_{1} f_{A_{1}}(u) \\
t_{B_{3}}(f(u) f(v))=t_{B_{3}}((g \circ h)(u)(g \circ h)(v)) & =t_{B_{3}}(g(h(u)) g(h(v))) \\
& =k_{4} t_{B_{2}}(h(u) h(v)) \\
& =k_{4} k_{2} t_{B_{1}}(u v) \\
f_{B_{3}}(f(u) f(v))=f_{B_{3}}((g \circ h)(u)(g \circ h)(v)) & =f_{B_{3}}(g(h(u)) g(h(v))) \\
& =k_{4} f_{B_{2}}(h(u) h(v)) \\
& =k_{4} k_{2} f_{B_{1}}(u v) .
\end{aligned}
$$

Thus there exists $\left(k_{3} k_{1}, k_{4} k_{2}\right)$ morphism $f$ from $G_{1}$ to $G_{3}$. Therefore $G_{1} \approx G_{2}$ and hence $\approx$ is transitive. So, the relation h-morphism is an equivalence relation in the collection of vague graphs.

Theorem 10 Let $G_{1}$ and $G_{2}$ be two vague graphs such that $G_{1}$ is $\left(k_{1}, k_{2}\right)$ vague morphism to $G_{2}$ for some non-zero $k_{1}$ and $k_{2}$. The image of strong edge in $G_{1}$ is strong edge in $G_{2}$ if and only if $k_{1}=k_{2}$.

Proof Let $(u, v)$ be strong edge in $G_{1}$ such that $h(u), h(v)$ is also strong edge in $G_{2}$. Now as $G_{1} \approx G_{2}$ we have

$$
\begin{aligned}
K_{2} t_{B_{1}}(u v)=t_{B_{2}}(h(u) h(v)) & =t_{A_{2}}(h(u) \wedge h(v)) \\
& =k_{1} t_{A_{1}}(u) \wedge k_{1} t_{A_{1}}(v) \\
& =k_{1} t_{B_{1}}(u v), \text { for all } u \in V_{1} .
\end{aligned}
$$


Hence, $k_{2} t_{B_{1}}(u v)=k_{1} t_{B_{1}}(u v)$, for all $u \in V_{1}$.

$$
\begin{aligned}
K_{2} f_{B_{1}}(u v)=f_{B_{2}}((h(u) h(v))) & =f_{A_{2}}(h(u) \vee h(v)) \\
& =k_{1} f_{A_{1}}(u) \vee k_{1} f_{A_{1}}(v) \\
& =k_{1} f_{B_{1}}(u v), \text { for all } u \in V_{1} .
\end{aligned}
$$

Hence $k_{2} f_{B_{1}}(u v)=k_{1} f_{B_{1}}(u v)$, for all $u \in V_{1}$.

The equations holds if and only if $k_{1}=k_{2}$.

Corollary 11 Let $G_{1}$ and $G_{2}$ be two vague graphs. Let $G_{1}$ be $\left(k_{1}, k_{2}\right)$ vague morphism to $G_{2}$. Let $G_{1}$ be strong. Then $G_{2}$ is strong if and only if $k_{1}=k_{2}$.

Theorem 12 If a vague graphs $G_{1}$ is co weak isomorphic to $G_{2}$ and if $G_{1}$ is regular then $G_{2}$ is regular also.

Proof As vague graph $G_{1}$ is co weak isomorphic to $G_{2}$, there exists a co weak isomorphism $h: G_{1} \rightarrow G_{2}$ which is bijective that satisfies $t_{A_{1}}(u) \leq t_{A_{2}}(h(u))$ and $f_{A_{1}}(u) \geq f_{A_{2}}(h(u)$ ), $t_{B_{1}}(u v)=t_{B_{2}}(h(u) h(v))$ and $f_{B_{1}}(u v)=f_{B_{2}}(h(u) h(v))$, for all $u, v \in V_{1}$. As $G_{1}$ is regular, for $u \in V, \sum_{u \neq v, v \in V_{1}} t_{B_{1}}(u v)=$ constant and $\sum_{u \neq v, v \in V_{1}} f_{B_{1}}(u v)=$ constant.

Now, $\sum_{h(u) \neq h(v)} t_{B_{2}}(h(u) h(v))=\sum_{u \neq v, v \in V_{1}} t_{B_{1}}(u v)=$ constant and $\sum_{h(u) \neq h(v)} f_{B_{2}}$ $(h(u) h(v))=\sum_{u \neq v, v \in V_{1}} f_{B_{1}}(u v)=$ constant. Therefore $G_{2}$ is regular.

Corollary 13 If a vague graph $G_{1}$ is co weak isomorphic to $G_{2}$ and if $G_{1}$ is strong, then $G_{2}$ need not be strong.

Theorem 14 Let $G_{1}$ and $G_{2}$ be two vague graphs. If $G_{1}$ is weak isomorphic to $G_{2}$ and if $G_{1}$ is strong then $G_{2}$ is strong also.

Proof As vague graph $G_{1}$ is weak isomorphic with $G_{2}$, there exists a weak isomorphism $h: G_{1} \rightarrow G_{2}$ which is bijective that satisfies $t_{A_{1}}(u)=t_{A_{2}}(h(u))$, $f_{A_{1}}(u)=f_{A_{2}}(h(u)), t_{B_{1}}(u v) \leq t_{B_{2}}(h(u) h(v))$ and $f_{B_{1}}(u v) \geq f_{B_{2}}(h(u) h(v))$. As $G_{1}$ is strong, $t_{B_{1}}(u v)=\min \left(t_{A_{1}}(u), t_{A_{1}}(v)\right)$ and $f_{B_{1}}(u v)=\max \left(f_{A_{1}}(u) f_{A_{1}}(v)\right)$. Now we have

$$
\begin{aligned}
t_{B_{2}}(h(u) h(v)) \geq t_{B_{1}}(u v) & =\min \left(t_{A_{1}}(u), t_{A_{1}}(v)\right) \\
& =\min \left(t_{A_{2}}(h(u)) t_{A_{2}}(h(v))\right) .
\end{aligned}
$$

By the definition, $t_{B_{2}}(h(u) h(v)) \leq \min \left(t_{A_{2}}(h(u)), t_{A_{2}}(h(v))\right)$. Therefore, $t_{B_{2}}(h(u) h(v))=$ $\min \left(t_{A_{2}}(h(u)), t_{A_{2}}(h(v))\right)$. Similarly,

$$
\begin{aligned}
f_{B_{2}}(h(u) h(v)) \leq f_{B_{1}}(u v) & =\max \left(f_{A_{1}}(u), f_{A_{1}}(v)\right) \\
& =\max \left(f_{A_{2}}(h(u)) f_{A_{2}}(h(v))\right) .
\end{aligned}
$$

By the definition, $\max \left(f_{A_{2}}(h(u)), f_{A_{2}}(h(v))\right) \leq f_{B_{2}}(h(u) h(v))$. Therefore, $f_{B_{2}}(h(u) h(v))=$ $\max \left(f_{A_{2}}(h(u)), f_{A_{2}}(h(v))\right)$. So, $G_{2}$ is strong.

Corollary 15 Let $G_{1}$ and $G_{2}$ be two vague graphs. If $G_{1}$ is weak isomorphic to $G_{2}$ and if $G_{1}$ is regular, then $G_{2}$ need not be regular. 
Theorem 16 If the vague graph $G_{1}$ is co weak isomorphic with a strong regular vague graph $G_{2}$, then $G_{1}$ is strong regular vague graph.

Proof As vague graph $G_{1}$ is co weak isomorphic with a vague graph $G_{2}$, there exists a co weak isomorphism $h: G_{1} \rightarrow G_{2}$ which is bijective that satisfies $t_{A_{1}}(u) \leq t_{A_{2}}(h(u)$ ), $f_{A_{1}}(u) \geq f_{A_{2}}(h(u)), t_{B_{1}}(u v)=t_{B_{2}}(h(u) h(v))$ and $f_{B_{1}}(u v)=f_{B_{2}}(h(u) h(v))$, for all $u, v \in V_{1}$. Now we have

$$
\begin{aligned}
t_{B_{1}}(u v)=t_{B_{2}}(h(u) h(v)) & =\min \left(t_{A_{2}}(h(u)), t_{A_{2}}(h(v))\right) \\
& \geq \min \left(t_{A_{1}}(u), t_{A_{1}}(v)\right) \\
f_{B_{1}}(u v)=f_{B_{2}}(h(u) h(v)) & =\max \left(f_{A_{2}}(h(u)), f_{A_{2}}(h(v))\right) \\
& \leq \max \left(f_{A_{1}}(u), f_{A_{1}}(v)\right) .
\end{aligned}
$$

But by definition $t_{B_{1}}(u v) \leq \min \left(t_{A_{1}}(u), t_{A_{1}}(v)\right)$ and $f_{B_{1}}(u v) \geq \max \left(f_{A_{1}}(u), f_{A_{1}}(v)\right)$. So, $t_{B_{1}}(u v)=\min \left(t_{A_{1}}(u), t_{A_{1}}(v)\right)$ and $f_{B_{1}}(u v)=\max \left(f_{A_{1}}(u), f_{A_{1}}(v)\right)$.

Therefore, $G_{1}$ is strong. Also for $u \in V_{1}, \sum_{u \neq v, v \in V_{1}} t_{B_{1}}(u v)=\sum t_{B_{2}}(h(u) h(v))=$ constant as $G_{2}$ is regular and $\sum_{u \neq v} f_{B_{1}}(u v)=\sum f_{B_{2}}(h(u) h(v))=$ constant as $G_{2}$ is regular. Therefore $G_{1}$ s regular.

Theorem 17 Let $G_{1}$ and $G_{2}$ be two isomorphic vague graphs, then $G_{1}$ is strong regular if and only if $G_{2}$ is strong regular.

Proof As a vague graph $G_{1}$ s isomorphic with vague graph $G_{2}$, there exists an isomorphism $h: G_{1} \rightarrow G_{2}$ which is bijective and satisfies $t_{A_{1}}(u)=t_{A_{2}}(h(u))$ and $f_{A_{1}}(u)=f_{A_{2}}(h(u))$, for all $u \in V_{1}$ and $t_{B_{1}}(u v)=t_{B_{2}}(h(u) h(v))$ and $f_{B_{1}}(u v)=f_{B_{2}}(h(u) h(v))$, for all $u v \in E_{1}$. Now, $G_{1}$ is strong if and only if $t_{B_{1}}(u v)=\min \left(t_{A_{1}}(u), t_{A_{1}}(v)\right)$ and $f_{B_{1}}(u v)=\max \left(f_{A_{1}}(u), f_{A_{1}}(v)\right)$ if and only if $t_{B_{2}}(h(u) h(v))=\min \left(t_{A_{2}}(h(u)), t_{A_{2}}(h(v))\right)$ and $f_{B_{2}}(h(u) h(v))=\max \left(f_{A_{2}}(h(u)), f_{A_{2}}(h(v))\right)$ if and only if $G_{2}$ is strong. $G_{1}$ is regular if and only if $\sum_{u \neq v, v \in V_{1}} t_{B_{1}}(u v)=$ constant, for all $u \in V_{1}$ and $\sum_{u \neq v, v \in V_{1}} f_{B_{1}}(u v)=$ constant, for all $u \in V_{1}$ if and only if $\sum_{h(u) \neq h(v), h(v) \in V_{2}} t_{B_{2}}(h(u) h(v))=$ constant and $\sum_{h(u) \neq h(v), h(v) \in V_{2}} f_{B_{2}}(h(u) h(v))=$ constant, for all $h(u) \in V_{2}$ if and only if $G_{2}$ is regular.

Theorem 18 A vague graphs $G_{1}$ is strong regular if and only if its complement vague graph $\bar{G}$ is strong regular vague graph also.

Proof The complement of a vague graph is defines as $t_{A_{1}}=\overline{t_{A_{1}}}, f_{A_{1}}=\overline{f_{A_{1}}}$, $\overline{t_{B}}(u v)=t_{A}(u) \wedge t_{A}(v)-t_{B}(u v)$ and $\overline{f_{B}}(u v)=f_{B}(u v)-f_{A}(u) \vee f_{A}(v) . \quad G$ is strong regular if and only if $t_{B}(u v)=\min \left(t_{A}(u), t_{A}(v)\right)$ and $f_{B}(u v)=\max \left(f_{A}(u), f_{A}(v)\right)$ if and only if $\overline{t_{B}}(u v)=t_{A}(u) \wedge t_{A}(v)-t_{B}(u v)=t_{B}(u v)-t_{B}(u v)=0 \quad$ and $\overline{f_{B}}(u v)=f_{B}(u v)-f_{A}(u) \vee f_{A}(v)=f_{B}(u v)-f_{B}(u v)=0$ if and only if $\sum \overline{t_{B}}(u v)=0$ and $\sum \overline{f_{B}}(u v)=0$ if and only if $\bar{G}$ is strong regular vague graph.

Definition 19 Let $G=(A, B)$ be a connected vague graph. $G$ is said to be a highly irregular vague graph if every vertex of $G$ is adjacent to vertices with distinct degrees.

Example 20 Consider a vague graph $G$ such that $V=\left\{v_{1}, v_{2}, v_{3}, v_{4}\right\}$ and $E=\left\{v_{1} v_{2}, v_{2} v_{3}, v_{3} v_{4}, v_{4} v_{1}\right\}$. By routine computations, we have $\operatorname{deg}\left(v_{1}\right)=(0.3,1.5)$, 
$\operatorname{deg}\left(v_{2}\right)=(0.2,1.3), \operatorname{deg}\left(v_{3}\right)=(0.4,1.2), \operatorname{deg}\left(v_{4}\right)=(0.5,1.4)$. We see that every vertex of $G$ is adjacent to vertices with distinct degrees. So, $G$ is highly irregular vague graph (see Fig. 3).

Theorem 21 For any two isomorphic highly irregular vague graphs, their order and size are same.

Proof If $h$ from $G_{1}$ to $G_{2}$ be an isomorphism between the highly irregular vague graphs $G_{1}$ and $G_{2}$ with the underlying sets $V_{1}$ and $V_{2}$ respectively then,

$$
\begin{aligned}
t_{A_{1}}(u) & =t_{A_{2}}(h(u)), f_{A_{1}}(u)=f_{A_{2}}(h(u)), \text { for all } u \in V, \\
t_{B_{1}}(u v) & =t_{B_{2}}(h(u) h(v)), f_{B_{1}}(u v)=f_{B_{2}}(h(u) h(v)), \text { for all } u, v \in V .
\end{aligned}
$$

So, we have

$$
\begin{aligned}
O\left(G_{1}\right) & =\left(\sum_{u_{1} \in V_{1}} t_{A_{1}}\left(u_{1}\right), \sum_{u_{1} \in V_{1}} f_{A_{1}}\left(u_{1}\right)\right)=\left(\sum_{u_{1} \in V_{1}} t_{A_{2}}\left(h\left(u_{1}\right)\right), \sum_{u_{1} \in V_{1}} f_{A_{2}}\left(h\left(u_{1}\right)\right)\right) \\
& =\left(\sum_{u_{2} \in V_{2}} t_{A_{2}}\left(u_{2}\right), \sum_{u_{2} \in V_{2}} f_{A_{2}}\left(u_{2}\right)\right)=O\left(G_{2}\right) \\
S\left(G_{1}\right) & =\left(\sum_{u_{1} v_{1} \in E_{1}} t_{B_{1}}\left(u_{1} v_{1}\right), \sum_{u_{1} v_{1} \in E_{1}} f_{B_{1}}\left(u_{1} v_{1}\right)\right) \\
& =\left(\sum_{u_{1}, v_{1} \in V_{1}} t_{B_{2}}\left(h\left(u_{1}\right) h\left(v_{1}\right)\right), \sum_{u_{1}, v_{1} \in V_{1}} f_{B_{2}}\left(h\left(u_{1}\right) h\left(v_{1}\right)\right)\right) \\
& =\left(\sum_{u_{2} v_{2} \in E_{2}} t_{B_{2}}\left(u_{2} v_{2}\right), \sum_{u_{2} v_{2} \in E_{2}} f_{B_{2}}\left(u_{2} v_{2}\right)\right)=S\left(G_{2}\right) .
\end{aligned}
$$

Theorem 22 If $G_{1}$ and $G_{2}$ are isomorphic highly irregular vague graphs then, the degrees of the corresponding vertices $u$ and $h(u)$ are preserved.

Proof If $h: G_{1} \rightarrow G_{2}$ is an isomorphism between the highly irregular vague graphs $G_{1}$ and $G_{2}$ with the underlying sets $V_{1}$ and $V_{2}$ respectively then, $t_{B_{1}}\left(u_{1} v_{1}\right)=t_{B_{2}}\left(h\left(u_{1}\right) h\left(v_{1}\right)\right)$ and $f_{B_{1}}\left(u_{1} v_{1}\right)=f_{B_{2}}\left(h\left(u_{1}\right) h\left(v_{1}\right)\right)$ for all $u_{1}, v_{1} \in V_{1}$. Therefore,

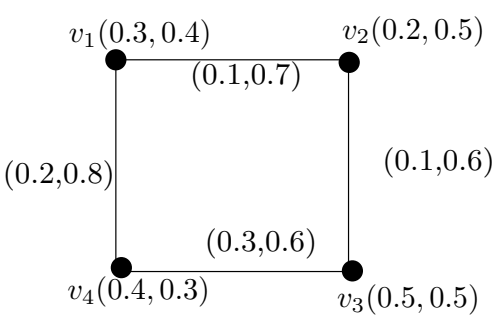

Fig. 3 A highly irregular vague graph 


$$
\begin{aligned}
& d_{t}\left(u_{1}\right)=\sum_{u_{1}, v_{1} \in V_{1}} t_{B_{1}}\left(u_{1} v_{1}\right)=\sum_{u_{1}, v_{1} \in V_{1}} t_{B_{2}}\left(h\left(u_{1}\right) h\left(v_{1}\right)\right)=d_{t}\left(h\left(u_{1}\right)\right) \\
& d_{f}\left(u_{1}\right)=\sum_{u_{1}, v_{1} \in V_{1}} f_{B_{1}}\left(u_{1} v_{1}\right)=\sum_{u_{1}, v_{1} \in V_{1}} f_{B_{2}}\left(h\left(u_{1}\right) h\left(v_{1}\right)\right)=d_{f}\left(h\left(u_{1}\right)\right)
\end{aligned}
$$

for all $u_{1} \in V_{1}$. That is, the degrees of the corresponding vertices of $G_{1}$ and $G_{2}$ are the same.

Definition 23 A vague graph $G$ is said to be

1. self complementary if $G \cong \bar{G}$,

2. self weak complementary if $G$ is weak isomorphic with $\bar{G}$.

Example 24 Let us consider, a vague graph $G=(A, B)$ where, the vertex set be $V=\left\{v_{1}, v_{2}, v_{2}\right\}$ and edge set is $\left\{v_{1} v_{2}, v_{2} v_{3}\right\}$. Obviously, the graph is self complementary (see Fig. 4). If identity bijective mapping is assumed, then $G$ and $\bar{G}$ are weak isomorphism.

Theorem 25 Let $\mathrm{G}$ be a self weak complementary highly irregular vague graph then,

$$
\begin{aligned}
& \sum_{u \neq v} t_{B}(u v) \leq \frac{1}{2} \sum_{u \neq v} \min \left(t_{A}(u), t_{A}(v)\right), \\
& \sum_{u \neq v} f_{B}(u v) \geq \frac{1}{2} \sum_{u \neq v} \max \left(f_{A}(u), f_{A}(v)\right) .
\end{aligned}
$$

Proof Let $G=(A, B)$ be a self weak complementary highly irregular vague graph of $G^{*}=(V, E)$. Then, there exists a weak isomorphism $h: G \rightarrow \bar{G}$ such that for all $u, v \in V$ we have $t_{A}(u)=\overline{t_{A}}(h(u))=t_{A}(h(u)), f_{A}(u)=\overline{f_{A}}(h(u))=f_{A}(h(u)) t_{B}(u v) \leq \overline{t_{B}}(h(u) h(v))$, $f_{B}(u v) \geq \overline{f_{B}}(h(u) h(v))$.

Using the definition of complement in the above inequality, for all $u, v \in V$ we have

$$
\begin{aligned}
& t_{B}(u v) \leq \overline{t_{B}}(h(u) h(v))=\min \left(t_{A}(h(u)), t_{A} h(v)\right)-t_{B}(h(u) h(v)) \\
& f_{B}(u v) \geq \overline{f_{B}}(h(u) h(v))=f_{B}(h(u) h(v))-\max \left(f_{A}(h(u)), f_{A}(h(v))\right. \\
& t_{B}(u v)+t_{B}(h(u) h(v)) \leq \min \left(t_{A}(h(u)), t_{A}(h(v))\right) \\
& f_{B}(u v)+f_{B}(h(u) h(v)) \geq \max \left(f_{A}(h(u)), f_{A}(h(v))\right) .
\end{aligned}
$$

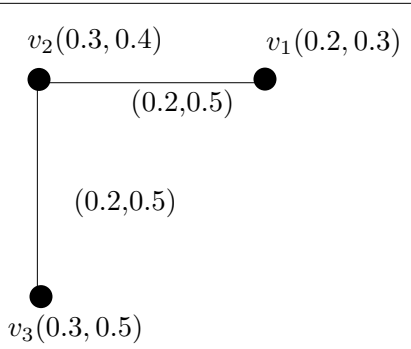

Fig. 4 An example of vague graph with self complementary and weak complementary 
So, $\sum_{u \neq v} t_{B}(u v)+\sum_{u \neq v} t_{B}(h(u) h(v)) \leq \sum_{u \neq v} \min \left(t_{A}(h(u)), t_{A}(h(v))\right)$ and $\sum_{u \neq v} f_{B}(u v)+$ $\sum_{u \neq v} f_{B}(h(u) h(v)) \geq \sum_{u \neq v} \max \left(f_{A}(h(u)), f_{A}(h(v))\right)$. Hence, $\quad 2 \sum_{u \neq v} t_{B}(u v) \leq \sum_{u \neq v}$ $\min \left(t_{A}(u), t_{A}(v)\right)$ and $2 \sum_{u \neq v} f_{B}(u v) \geq \sum_{u \neq v} \max \left(f_{A}(u), f_{A}(v)\right)$. Now we have $\sum_{u \neq v} t_{B}(u v) \leq \frac{1}{2} \sum_{u \neq v} \min \left(t_{A}(u), t_{A}(v)\right)$ and $\sum_{u \neq v} f_{B}(u v) \geq \frac{1}{2} \sum_{u \neq v} \max \left(f_{A}(u), f_{A}(v)\right)$.

Definition 26 Let $G=(A, B)$ be a vague graph. The $\mu$-complement of $G$ is defined as $G^{\mu}=\left(A, B^{\mu}\right)$ where $B^{\mu}=\left(t_{B}^{\mu}, f_{B}^{\mu}\right)$ and

$$
\begin{array}{r}
t_{B}^{\mu}(u v)=\left\{\begin{array}{cc}
t_{A}(u) \wedge t_{A}(v)-t_{B}(u v) & \text { if } t_{B}(u v)>0 \\
0 & \text { if } t_{B}(u v)=0
\end{array}\right. \\
f_{B}^{\mu}(u v)=\left\{\begin{array}{cc}
f_{B}(u v)-f_{A}(u) \vee f_{A}(v) & \text { if } f_{B}(u v)>0 \\
0 & \text { if } f_{B}(u v)=0
\end{array}\right.
\end{array}
$$

Example 27 Let us consider a vague graph $G=(A, B)$ where the vertex set is $V=\left\{v_{1}, v_{2}, v_{3}\right\}$ and edge set is $E=\left\{v_{1} v_{2}, v_{2} v_{3}, v_{1} v_{3}\right\}$ (see Fig. 5).

Theorem 28 The $\mu$-complement of a highly irregular vague graph need not be highly irregular.

Proof To every vertex, the adjacent vertices with distinct degrees or the non-adjacent vertices with distinct degrees may happen to be adjacent vertices with same degrees. This contradicts the definition of highly irregular vague graph.

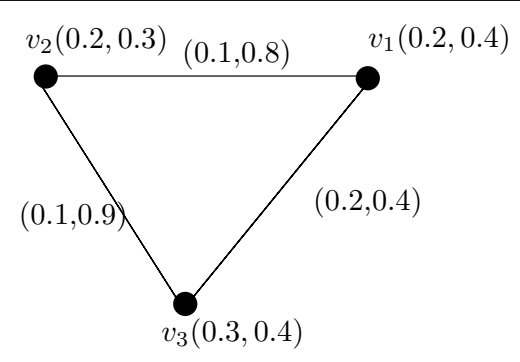

a

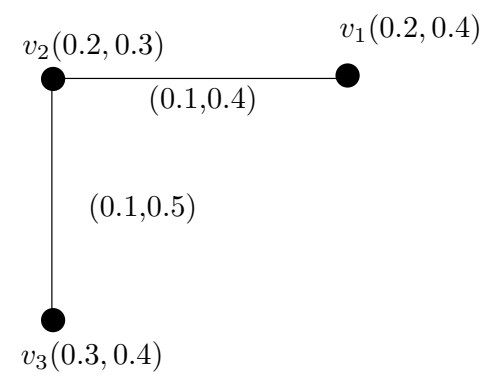

b

Fig. $5 \mu$-Complement of vague graphs. a Vague graph $G$. $\mathbf{b} \mu$-Complement of $G$ 
Theorem 29 Let $G_{1}$ and $G_{2}$ be two highly irregular vague graphs. If $G_{1}$ and $G_{2}$ are isomorphic, then $\mu$-complement of $G_{1}$ and $G_{2}$ are isomorphic also and vice versa.

Proof Assume that $G_{1}$ and $G_{2}$ are isomorphic, there exists a bijective map $h: V_{1} \rightarrow V_{2}$ satisfying $t_{A_{1}}(u)=t_{A_{2}}(h(u)), \quad f_{A_{1}}(u)=f_{A_{2}}(h(u))$, for all $u \in V_{1}$ and $t_{B_{1}}(u v)=t_{B_{2}}(h(u) h(v)), f_{B_{1}}(u v)=f_{B_{2}}(h(u) h(v))$, for all $u v \in E_{1}$. By the definition of $\mu-$ complement we have $t_{B_{1}}^{\mu}(u v)=\min \left(t_{A_{1}}(u), t_{A_{1}}(v)\right)-t_{B_{1}}(u v)=\min \left(t_{A_{2}}(h(u)), t_{A_{2}}(h(v))\right)$ $-t_{B_{2}}(h(u) h(v)), \quad f_{B_{1}}^{\mu}(u v)=f_{B_{1}}(u v)-\max \left(f_{A_{1}}(u), f_{A_{1}}(v)\right)=f_{B_{2}}(h(u) h(v))-\max \left(f_{A_{2}}(h(u))\right.$, $\left.f_{A_{2}}(h(v))\right)$, for all $u v \in E_{1}$. Hence, $G_{1}^{\mu} \cong G_{2}^{\mu}$. The proof of the converse part is straight forward.

\section{Conclusion}

It is well known that graphs are among the most ubiquitous models of both natural and human-made structures. They can be used to model many types of relations and process dynamics in computer science, physical, biological and social systems. In this paper, we introduced the notion of vague h-morphism on vague graphs and studied the action of vague $h$-morphism on vague strong regular graphs. We defined $\mu$-complement of highly irregular vague graphs and investigated its properties.

Authors' contributions

The authors contributed equally to each parts of the paper. All authors read and approved the final manuscript.

\section{Author details}

${ }^{1}$ Young Researchers and Elite Club, Central Tehran Branch, Islamic Azad University, Tehran, Iran. ${ }^{2}$ Department of Mathematics, Indian Institute of Information Technology, Nagpur 440006, India. ${ }^{3}$ Department of Applied Mathematics with Oceanology and Computer Programming, Vidyasagar University, Midnapore 721102, India. ${ }^{4}$ Department of Mathematics, Shahid Beheshti University, Tehran, Iran.

\section{Acknowledgements}

The authors are grateful to the Editor in Chief and honorable reviewers of the journal "Springer Plus" for their suggestions to improve the quality and representation of the paper. The authors are also grateful to the Head Master of Joykrishnapur High School (H.S.), Mr. Madhusudan Bhaumik for his help.

\section{Competing interests}

The authors claim that they have no competing interests.

Received: 17 December 2015 Accepted: 21 July 2016

Published online: 02 August 2016

\section{References}

Akram M, Gani N, Saeid AB (2014) Vague hypergraphs. J Intell Fuzzy Syst 26:647-653

Boorzooei RA, Rashmanlou H, Samanta S, Pal M (2016a) New concepts of vague competition graphs. J Intell Fuzzy Syst 31:69-75

Boorzooei RA, Rashmanlou H, Samanta S, Pal M (2016b) Regularity of vague graphs. J Intell Fuzzy Syst 30:3681-3689 Gau WL, Buehrer DJ (1993) Vague sets. IEEE Trans Syst Man Cybern 23(2):610-614

Kauffman A (1973) Introduction a la Theorie des Sous-Emsembles Flous, Masson et Cie, 1

Mordeson JN, Nair PS (2001) Fuzzy graphs and fuzzy hypergraphs, 2nd edn. Physica, Heidelberg

Pramanik T, Samanta S, Pal M (2016a) Interval-valued fuzzy planar graphs. Int J Mach Learn Cybern 7:653-664

PramanikT, Samanta S, Sarkar B, Pal M (2016b) Fuzzy phi-tolerance competition graphs. Soft Comput. doi:10.1007/ s00500-015-2026-5

Ramakrishna N (2009) Vague graphs. Int J Comput Cogn 7:51-58

Rashmanlou H, Pal M (2013a) Balanced interval-valued fuzzy graph. J Phys Sci 17:43-57

Rashmanlou H, Pal M (2013b) Some properties of highly irregular interval-valued fuzzy graphs. World Appl Sci J 27(12):1756-1773

Rashmanlou H, Samanta S, Borzooei RA, Pal M (2015a) A study on bipolar fuzzy graphs. J Intell Fuzzy Syst 28:571-580 Rashmanlou H, Samanta S, Pal M, Borzooei RA (2015b) A study on bipolar fuzzy graphs. J Intell Fuzzy Syst. doi:10.3233/ IFS-141333 
Rashmanlou H, Samanta S, Pal M, Borzooei RA (2015c) Bipolar fuzzy graphs with categorical properties. Int I Intell Comput Syst 8(5):808-818

Rosenfeld A (1975) Fuzzy graphs. In: Zadeh LA, Fu KS, Shimura M (eds) Fuzzy Sets and Their Applications. Academic Press, New York, pp 77-95

Samanta S, Pal M (2011a) Fuzzy threshold graphs. CiiT Int J Fuzzy Syst 3(12):360-364

Samanta S, Pal M (2011 b) Fuzzy tolerance graphs. Int J Latest Trends Math 1(2):57-67

Samanta S, Pal M (2012a) Irregular bipolar fuzzy graphs. Int J Appl Fuzzy Sets 2:91-102

Samanta S, Pal M (2012b) Bipolar fuzzy hypergraphs. Int J Fuzzy Logic Syst 2(1):17-28

Samanta S, Pal M (2013) Fuzzy k-competition graphs and p-competition fuzzy graphs. Fuzzy Eng Inf 5(2):191-204

Samanta S, Pal M (2014) Some more results on bipolar fuzzy sets and bipolar fuzzy intersection graphs. J Fuzzy Math 22(2):253-262

Samanta S, Pal M (2015) Fuzzy planar graphs. IEEE Trans Fuzzy Syst 23(6):1936-1942

Samanta S, Pal M, Akram M (2014a) m-Step fuzzy competition graphs. J Appl Math Comput. doi:10.1007/ S12190-014-0785-2

Samanta S, Pal M, Pal A (2014b) New concepts of fuzzy planar graph. Int J Adv Res Artif Intell 3(1):52-59

Samanta S, Pal M, Pal A (2014c) New concepts of fuzzy planar graph. Int J Adv Res Artif Intell 3(1):52-59

Samanta S, Pal M, Pal A (2014d) Some more results on fuzzy k-competition graphs. Int J Adv Res Artif Intell 3(1):60-67

Samanta S, Pal M, Rashmanlou H, Borzooei RA (2016) Vague graphs and strengths. J Intell Fuzzy Syst 30:3675-3680

Sunitha MS, Vijayakumar A (2002) Complement of a fuzzy graph. Indian J Pure Appl Math 33(9):1451-1464

\section{Submit your manuscript to a SpringerOpen ${ }^{\odot}$ journal and benefit from:}

- Convenient online submission

\section{- Rigorous peer review}

- Immediate publication on acceptance

- Open access: articles freely available online

- High visibility within the field

- Retaining the copyright to your article

Submit your next manuscript at $>$ springeropen.com 